\title{
25 Research Square \\ Systemic Inflammatory Score Predicts Overall Survival In Patients With Cervical Cancer
}

\section{$\mathrm{Mu} \mathrm{Xu}$}

Fujian Maternity and Child health hospital

\section{Qibin Wu}

Fujian Maternity and Child Health Hospital

\section{Liangzhi Cai}

Fujian Maternity and Child health hospital

\section{Xiaoqi Sun}

Fujian Maternity and Children Health Hospital

\section{Xiaoyan Xie}

Fujian Maternity and Children Health Hospital

Pengming Sun ( $\nabla$ sunfemy@hotmail.com )

Fujian Maternity and Children Health Hospital https://orcid.org/0000-0002-5072-6091

\section{Research article}

Keywords: Cervical cancer, Systemic inflammation score, Lymphocyte-to- monocyte ratio, Albumin, Prognosis

Posted Date: December 1st, 2020

DOl: https://doi.org/10.21203/rs.3.rs-115868/v1

License: (c) (i) This work is licensed under a Creative Commons Attribution 4.0 International License.

Read Full License 


\section{Abstract}

Background To evaluate the prognostic value of the systemic inflammatory score (SIS) in cervical cancer patients.

Methods The optimal cutoff values for inflammatory biomarkers were calculated by X-tile software. The prognostic factors were investigated using univariate and multivariate Cox analyses. Time-dependent receiver operating characteristic (time-ROC) analysis and the concordance index (C-index) were used to compare the prognostic impact of factors.

Results In total, 264 patients with cervical cancer were included in the study. The optimal cutoff value for lymphocyte-to-monocyte ratio (LMR) was 4.1. In multivariate analysis, FIGO stage, lymphovascular invasion, lymph node metastasis, preoperative serum albumin (Alb), and LMR were independent prognostic factors $(P<0.05)$. Then, we combined preoperative Alb and LMR to establish the SIS. Multivariate analysis showed that the SIS was an independent factor that affected survival $(P<0.05)$. When stratified by FIGO stage, significant differences in survival were also found for patients with different SISs $(P<0.05)$. When the SIS and FIGO stage were combined, the time-ROC curve was superior to that of FIGO stage only. The C-index of the model combining the SIS and FIGO stage was $0.786(95 \% \mathrm{CI}$ $0.699-0.873)$, which was significantly higher than that of the model with FIGO stage only $(0.676,95 \% \mathrm{Cl}$ $0.570-0.782, P=0.0049)$.

Conclusions The preoperative SIS is a simple and useful prognostic factor for postoperative survival in patients with cervical cancer. It might assist in the identification of high-risk patients among patients with the same FIGO stage.

\section{Background}

Cervical cancer (CC) remains the most common gynaecologic malignancy in females worldwide [1-2]. The postoperative recurrence and metastasis are the main causes of death in CC [3-4]. The International Federation of Gynaecology and Obstetrics (FIGO) staging system is the main criterion used to predict the prognosis of patients with CC so far. However, the prognosis varies in CC patients with the same FIGO stage. Some patients with early stage disease experience recurrence in a short time after surgery, leading to poor prognosis [5]. Therefore, it is necessary to find more potential biomarkers in clinical practice to improve prognostic prediction.

Previous studies showed inflammation affected cancer pathogenesis and progression [6-7]. Systemic inflammatory biomarkers, such as the lymphocyte-to-monocyte ratio (LMR), neutrophil-to-lymphocyte ratio (NLR), and platelet-to-lymphocyte ratio (PLR), have been reported as prognostic factors in various tumours [8-10]. The levels of serum albumin (Alb) before operation have also been reported as an independent predictor of prognosis in patients with malignancies [11]. These biomarkers can be detected in an easy and convenient way. Recently, the systemic inflammatory score (SIS), based on the level of preoperative Alb and LMR, has been found to be prognostic factor in some cancers [12-13]. However, the 
effect of SIS on the prognosis of CC patients remains unknown. Therefore, we aimed to assess the prognostic value of the SIS for patients with $\mathrm{CC}$ who underwent radical surgery.

\section{Methods \\ Patients}

A total of 264 patients with cervical cancer undergoing radical resection at the Fujian Provincial Maternity and Children's Hospital from January 2014 to December 2017 were recruited. The following inclusion criteria were applied: (1) histologically confirmed cervical cancer; (2) FIGO stage (2009) IB-IIA with no evidence of tumours invading adjacent organs or distant metastasis; and (3) radical hysterectomy with pelvic lymphadenectomy. The study excluded patients who had distant metastasis in the liver, lung, or peritoneum/pelvic cavity diagnosed before or during the operation; who underwent preoperative neoadjuvant radiotherapy and/or chemotherapy; who had active infection or inflammatory diseases within a month before blood examination; or who had incomplete/inaccurate medical records. The FIGO 2009 clinical staging system was used for tumour staging. This retrospective study was approved by the Ethics Committee of the Fujian Provincial Maternity and Children's Hospital. Written informed consent was obtained from all participants.

\section{Definition of systemic inflammatory biomarkers}

The blood testing was carried out within 1 week before surgery, including the neutrophil count, lymphocyte count, platelet count, and Alb level. LMR was defined as the total number of lymphocytes divided by the total number of monocytes. NLR and PLR were defined as the total number of neutrophils or platelets divided by the total number of lymphocytes. The cutoff value of Alb was $40 \mathrm{~g} / \mathrm{l}$ [12-13]. The optimal cutoff values for LMR, NLR and PLR were calculated by the X-tile software (Yale University, New Haven, CT, USA) [14].

\section{Followup}

Patients underwent follow-up examinations every 3 months for years 1 and 2, then every 6 months during years 3-5. The final follow-up evaluation was conducted in December 2019. The median follow-up period was 44 months (range 3-72 months). The main examinations included physical examination, vaginal examination, laboratory testing (including cancer antigen 125 (Ca125), Squamous cell carcinoma antigen (SCC)), chest radiography, and pelvic ultrasonography. The lung computed tomography (CT) or pelvic magnetic resonance imaging (MRI) scan was performed when necessary. Overall survival (OS) was defined as the time from surgery to the date of death or to the last follow-up.

\section{Statistical analysis}

Statistical analyses were performed using SPSS for Windows version 18.0 (SPSS Inc., Chicago, IL, USA) and $\mathrm{R}$ ver. 3.6.2 (R Foundation for Statistical Computing, Vienna, Austria). Categorical and continuous variables were compared using a chi-square test or Fisher's exact test and a $t$ test, respectively. The 
survival curves were calculated by the Kaplan-Meier method, and differences between them were examined using the log-rank test. Variables associated with $P<0.05$ in univariate analysis were then included in the multivariate Cox regression analysis. The time-dependent receiver operating characteristic (time-ROC) curves, which was an extension of the ROC curve [15], were used to compare the prognostic abilities of the scores. Model performance was then assessed by Concordance indices (C-index). The time-ROC analysis and C-index were performed respectively by using the R packages "rms" and "time ROC". All tests were 2-sided, and $\mathrm{P}<0.05$ indicated that the difference was statistically significant.

\section{Results}

\section{Patient characteristics}

The optimal cutoff levels for LMR, NLR and PLR were 4.1, 1.8 and 132.8, respectively, which were calculated by X-tile software (Supplemental Fig. 1).

In total, 264 patients were included in the study. The clinicopathological features of the patients were shown in Table 1. The median age of the patients was 47 years (24-71 years). Based on the FIGO staging system, 177 (67.0\%), 37 (14.1\%), and 50 (18.9\%) of the patients had FIGO stage Ib1, Ib2, and Ila disease, respectively. According to the cutoff value, 120 (45.5\%) patients had a high LMR, 144 (54.5\%) had a low LMR, 133 (50.4\%) had a high NLR, 131 (49.6\%) had a low NLR, 128 (48.5\%) had a high PLR, 136 (51.5\%) had a low PLR, 155 (58.7\%) had a high Alb and 109 (41.3\%) had a low Alb (Table 1). 
Table 1

Correlations between clinicopathologic variables and OS in univariate and multivariate analysis

\begin{tabular}{|c|c|c|}
\hline \multirow{2}{*}{$\begin{array}{l}\text { Clinicopathological } \\
\text { features }\end{array}$} & \multirow{2}{*}{$\begin{array}{l}\text { No. of } \\
\text { patients }\end{array}$} & Univariate analysis \\
\hline & & HR $(95 \% \mathrm{Cl})$ \\
\hline \multicolumn{3}{|l|}{ Age $(y)$} \\
\hline$<45$ & 106 & Reference \\
\hline$\geq 45$ & 158 & $1.332(0.564-3.145$ \\
\hline
\end{tabular}

FIGO stage

0.008

0.022

IB1

177

Reference

Reference

IB2

37

2.935(0.982-8.77)

$0.232(0.076-$

0.709 )

Ila

50

4.146(1.642-

10.467)

0.313(0.099-

0.984)

\section{Surgery}

Open

134

130

Reference

Lap

.

0.484(0.565-3.320)

Histological type

0.658

SCC

203 Reference

AC

46

0.751(0.221-2.55)

Others

15

1.726(0.399-7.461)

Lymphovascular

invation

No

182

Reference

Reference

Yes

82

5.697(2.34-13.875)

3.01(1.045-8.671)

Depth of invasion

0.011

0.815

$<1 / 2$

140

Reference

Reference

$\geq 1 / 2$

124

3.337(1.315-8.463)

Lymph node metastasis

0.002

0.024

No

228

Reference

Reference

Abbreviations: OS, overall survival; Alb, albumin; LMR, lymphocyte-to- monocyte ratio; NLR, neutrophilto-lymphocyte ratio; PLR, platelet-to-lymphocyte ratio; SCC, squamous cell carcinoma; AC, adenocarcinoma. 


\begin{tabular}{|c|c|c|c|c|c|}
\hline \multirow{2}{*}{$\begin{array}{l}\text { Clinicopathological } \\
\text { features }\end{array}$} & \multirow{2}{*}{$\begin{array}{l}\text { No. of } \\
\text { patients }\end{array}$} & \multicolumn{2}{|l|}{ Univariate analysis } & \multicolumn{2}{|c|}{ Multivariate analysis } \\
\hline & & $\mathrm{HR}(95 \% \mathrm{Cl})$ & $\mathbf{P}$ & $\mathrm{HR}(95 \% \mathrm{Cl})$ & $\mathbf{P}$ \\
\hline Yes & 36 & $3.901(1.653-9.209)$ & & $\begin{array}{l}0.359(0.158- \\
0.703)\end{array}$ & \\
\hline $\operatorname{Alb}(g / l)$ & & & 0.026 & & 0.039 \\
\hline$<40$ & 109 & Reference & & Reference & \\
\hline$\geq 40$ & 155 & $0.194(0.07-0.499)$ & & $0.123-0.945$ & \\
\hline LMR & & & 0.014 & & 0.020 \\
\hline$<4.1$ & 144 & Reference & & Reference & \\
\hline$\geq 4.1$ & 120 & $0.111(0.026-0.471)$ & & $\begin{array}{l}0.167(0.037- \\
0.755)\end{array}$ & \\
\hline NLR & & & 0.019 & & 0.400 \\
\hline$<1.8$ & 131 & Reference & & Reference & \\
\hline$\geq 1.8$ & 133 & $3.055(1.204-7.752)$ & & $\begin{array}{l}1.542(0.562- \\
4.231)\end{array}$ & \\
\hline PLR & & & 0.024 & & 0.135 \\
\hline$<132.8$ & 136 & Reference & & Reference & \\
\hline$\geq 132.8$ & 128 & $2.783(1.143-6.778)$ & & $\begin{array}{l}2.134(0.789- \\
5.771)\end{array}$ & \\
\hline $\begin{array}{l}\text { Abbreviations: OS, o } \\
\text { to-lymphocyte ratio; } \\
\text { adenocarcinoma. }\end{array}$ & $\begin{array}{l}\text { urvival; A } \\
\text { atelet-to-I }\end{array}$ & $\begin{array}{l}\text { albumin; LMR, lymph } \\
\text { iphocyte ratio; SCC, s }\end{array}$ & $\begin{array}{l}\text { te-to- } n \\
\text { amous }\end{array}$ & $\begin{array}{l}\text { nocyte ratio; } N \\
\text { ll carcinoma; } A\end{array}$ & rophil- \\
\hline
\end{tabular}

\section{Survival analysis}

The 5-year OS rate was $89.1 \%$ in all patients. Univariate analysis showed that FIGO stage, lymphovascular invasion, deep stromal invasion, lymph node metastasis, preoperative Alb, and inflammatory indicators including LMR, NLR, and PLR were associated with OS (all $P<0.05$ ). Further multivariate Cox regression analysis showed that FIGO stage, lymphovascular invasion, lymph node metastasis, preoperative Alb and LMR were independent factors for prognosis (Table 1).

\section{Correlations between clinicopathological features and the SIS}

As Alb level ( $\geq 40 \mathrm{~g} / \mathrm{l})$ and $L M R \geq 4.1$ were associated with good OS (both $P<0.05$, Table 1 ), the new indicator SIS was then established based on preoperative Alb and LMR, and the patients were divided into three subgroups. Patients with both decreased serum albumin and decreased LMR $(<40 \mathrm{~g} / \mathrm{l}$ and $<4.1$, respectively) were assigned a score of 2; patients with either decreased serum albumin or decreased LMR 
were assigned a score of 1 ; and patients with both elevated serum albumin and elevated LMR ( $\geq 40 \mathrm{~g} / \mathrm{l}$ and $\geq 4.1$, respectively) were assigned a score of 0 .

The relationships between clinicopathological factors and the SIS are shown in Table 2. The results showed that higher FIGO stage, lymphovascular invasion, deep stromal invasion, and lymph node metastasis were significantly associated with higher SISs (all $P<0.05$, Table 2). 
Table 2

Relationship between the SIS and clinicopathological variables of patients

Clinicopathological

features

Age (y)

$<45$

$\geq 45$

FIGO stage

IB1

IB2

Ila

Histological type

SCC

AC

Others

Lymphovascular invation

No

Yes

Depth of invasion

$<1 / 2$

$\geq 1 / 2$

Lymph node metastasis

No

Yes

NLR

$<1.8$

$\geq 1.8$

PLR
SIS

$P$ value

$\begin{array}{lll}0 & 1 & 2\end{array}$

2

0.474

\begin{tabular}{lll}
$35(39.8 \%)$ & $49(43.8 \%)$ & $22(34.4 \%)$ \\
\hline $53(60.2 \%)$ & $63(56.2 \%)$ & $42(65.6 \%)$
\end{tabular}

0.020

\begin{tabular}{lll}
\hline $67(76.1 \%)$ & $76(67.8 \%)$ & $34(53.1 \%)$ \\
\hline $8(9.1 \%)$ & $15(13.4 \%)$ & $14(21.9 \%)$ \\
\hline $13(14.8 \%)$ & $21(18.8 \%)$ & $16(25.0 \%)$
\end{tabular}

0.493

$70(79.5 \%)$

$82(73.2 \%)$

$51(79.7 \%)$

$15(17.1 \%)$

23(20.5\%)

$8(12.5 \%)$

$3(3.4 \%)$

$7(6.3 \%)$

$5(7.8 \%)$

$<0.001$

$75(85.2 \%)$

73(65.2\%)

$34(53.1 \%)$

13(14.8\%)

$39(34.8 \%)$

$30(46.9 \%)$

$<0.001$

$62(70.5 \%) \quad 54(48.2 \%) \quad 24(37.5 \%)$

$26(29.5 \%)$

$58(51.8 \%)$

$40(62.5 \%)$

$<0.001$

$86(97.7 \%)$

94(83.9\%)

$48(75.0 \%)$

$2(2.3 \%)$

$18(16.1 \%)$

$16(25.0 \%)$

0.002

\begin{tabular}{lll}
$56(63.6 \%)$ & $52(46.4 \%)$ & $23(35.9 \%)$ \\
\hline $32(36.4 \%)$ & $60(53.6 \%)$ & $41(64.1 \%)$
\end{tabular}

$60(53.6 \%)$

$41(64.1 \%)$

Abbreviations: SIS, Systemic Inflammatory Score; SCC, squamous cell carcinoma; AC, adenocarcinoma; NLR, neutrophil-to-lymphocyte ratio; PLR, platelet-to-lymphocyte ratio. 


\begin{tabular}{|llll|}
\hline \multirow{2}{*}{$\begin{array}{l}\text { Clinicopathological } \\
\text { features }\end{array}$} & \multicolumn{2}{c|}{ SIS } & P value \\
\cline { 2 - 4 } & $\mathbf{0}$ & $\mathbf{1}$ & $\mathbf{2}$ \\
\hline$<132.8$ & $56(63.6 \%)$ & $55(49.1 \%)$ & $25(39.1 \%)$ \\
\hline$\geq 132.8$ & $32(36.4 \%)$ & $57(50.9 \%)$ & $39(0.9 \%)$ \\
\hline $\begin{array}{l}\text { Abbreviations: SIS, Systemic Inflammatory Score; SCC, squamous cell carcinoma; AC, } \\
\text { adenocarcinoma; NLR, neutrophil-to-lymphocyte ratio; PLR, platelet-to-lymphocyte ratio. }\end{array}$ \\
\hline Influence Of the SIS On OS
\end{tabular}

Kaplan-Meier curves showed significant differences in the 5-year OS among three groups according to the SIS (SIS $=0: 98.1 \%$, SIS $=1: 72.5 \%$, SIS $=2: 72.5 \%$, all $P<0.05$, Fig. 1). Furthermore, multivariate analyses revealed that FIGO stage, lymphovascular invasion, deep stromal invasion and SIS were significantly associated with OS (all $P<0.05$, Table 3 ). In addition, when stratified by FIGO stage, there were also significant differences in OS with a SIS of 0,1 and 2 in the stage Ib and II subgroups (all $P<$ 0.05, Fig. 2). 
Table 3

Multivariate analysis of clinicopathologic variables in relation to OS in patients

\begin{tabular}{|c|c|c|c|}
\hline \multirow{2}{*}{$\begin{array}{l}\text { Clinicopathological } \\
\text { features }\end{array}$} & \multirow{2}{*}{$\begin{array}{l}\text { No. of } \\
\text { patients }\end{array}$} & \multicolumn{2}{|l|}{ os } \\
\hline & & $\mathrm{HR}(95 \% \mathrm{Cl})$ & $P$ value \\
\hline FIGO stage & & & 0.027 \\
\hline IB1 & 177 & Reference & \\
\hline IB2 & 37 & $1.285(0.375-4.403)$ & \\
\hline Ila & 50 & $4.053(1.335-12.310)$ & \\
\hline Lymphovascular invation & & & 0.033 \\
\hline No & 182 & Reference & \\
\hline Yes & 82 & $3.092(1.093-8.741)$ & \\
\hline Depth of invasion & & & 0.906 \\
\hline$<1 / 2$ & 140 & Reference & \\
\hline$\geq 1 / 2$ & 124 & $0.933(0.295-2.956)$ & \\
\hline Lymph node metastasis & & & 0.025 \\
\hline No & 228 & Reference & \\
\hline Yes & 36 & $2.116(1.014-5.746)$ & \\
\hline NLR & & & 0.352 \\
\hline$<1.8$ & 131 & Reference & \\
\hline$\geq 1.8$ & 133 & $1.632(0.582-4.578)$ & \\
\hline PLR & & & 0.162 \\
\hline$<132.8$ & 136 & Reference & \\
\hline$\geq 132.8$ & 128 & $2.062(0.747-5.90)$ & \\
\hline SIS & & & 0.008 \\
\hline 0 & 88 & Reference & \\
\hline 1 & 112 & $3.639(0.421-31.433)$ & \\
\hline 2 & 64 & $11.733(1.410-97.616)$ & \\
\hline
\end{tabular}

\section{The predictive value of SIS on prognosis}


Moreover, the predictive accuracy of the model combining the SIS and FIGO stage (SFGO) was compared with that of the model containing FIGO stage only by establishing time-ROC curves (Fig. 3). As the results showed, the time-ROC curve of the model combining the SIS and FIGO stage was superior to that of the model containing FIGO stage only. In addition, the C-index of the model combining the SIS and FIGO stage was $0.786(95 \% \mathrm{Cl}: 0.699-0.873)$, which was significantly higher than that of the model with only FIGO stage $(0.676,95 \% \mathrm{Cl}: 0.570-0.782 ; P=0.0049)$.

\section{Discussion}

Although FIGO stage is the most important clinical prognostic indicator for cervical cancer patients, many patients with the same FIGO stage have different treatment outcomes due to tumour heterogeneity [5]. Therefore, it is necessary to find other indicators to assist in predicting the prognosis of cervical cancer. As Virchow originally made links between cancer and inflammation in 1863, more and more investigations have revealed that inflammation plays an important role in tumour progression and metastasis [7, 17]. Previous studies have also shown that inflammatory indicators significantly influence the prognosis of gynaecologic cancers [18-20]. Recently, the SIS, based on the combination of serum albumin and LMR, was reported to have prognostic value in renal cell carcinoma and colorectal cancer $[12,13]$. Compared to other single nutritional or inflammatory makers, the SIS may be a better indicator because of considering the influences of both nutritional condition and inflammation on the prognosis of tumours. However, the influence of the SIS on the prognosis of cervical cancer remains unclear.

In this study, the cutoff value of LMR was 4.1, as calculated by X-tile. Multivariable analysis demonstrated that LMR $\geq 4.1$ and $\mathrm{Alb} \geq 40 \mathrm{~g} / \mathrm{I}$ were associated with favourable OS. Additionally, the SIS was associated with a number of variables that were previously shown to be predictive of poor outcomes. The SIS was also an independent prognostic factor according to multivariable analysis.

LMR consists of lymphocytes and monocytes. As the basic components of the immune system, lymphocytes can stimulate the proliferation of cytotoxic T cells by secreting cytokines such as IFN- $\gamma$ and TNF-a. As a result, the tumour cell proliferation, invasion and metastasis are inhibited, resulting in cytotoxic death and improving the prognosis $[21,22]$. Moreover, the lymphocytes transform into tumourinfiltrating lymphocytes when migrate to the tumour microenvironment, resulting in anti-tumour activity and the inhibition of angiogenesis to improve the outcomes in cancers [23]. Thus, a decline in lymphocytes predicted poor outcomes in patients with tumour [24]. In addition, monocytes can be recruited to tumour tissues and differentiate into tumour-associated macrophages (TAMs). Then, TAMs release epidermal growth factor and angiogenic factors to promote tumour cell proliferation and migration [25-27]. Chen et al [28] demonstrated the prognostic value of preoperative LMR in predicting the survival of 485 patients with FIGO stage IB1-IIA cervical cancer. Therefore, LMR plays an important role in the prognosis of cervical cancer. Alb is closely related to nutritional status in patients, which is a good indicator of immune status. Zheng found that hypoalbuminemia was associated with poor survival in 798 patients with early-stage cervical cancer [29], which was also observed in our study. Additionally, the SIS was also significantly influenced by prognosis when stratified by FIGO stage. In agreement with 
previous findings, we demonstrated that high SIS was an independent predictor of diminished survival for CC patients.

Recently, time-ROC analysis has been used in some studies [14]. It's a method that applies ROC curve analysis to time-dependent variables. The advantage of this method is that it enables the analysis of survival data with censoring using ROC curves, which is a popular method of determining sensitivity and specificity. What's more, the significant difference among prognostic factors can be assessed visually. In our study, we created a new prognostic model by combining the SIS and FIGO stage. As the result showed, the time-ROC curve and the C-index of the new model combining the SIS with FIGO stage were significantly higher than the model with traditional FIGO stage only, which suggested that the predictive accuracy of the new model was better. In other words, the SIS could improve the accuracy of the prognostic assessment in patients with cervical cancer. Therefore, the SIS can be used as a supplement to FIGO stage in preoperative risk stratification to improve the prediction of clinical outcomes.

However, there were also some limitations in the study. First, as a retrospective, single-center study, the possibility of selection bias is inevitable. Therefore, a large-scale prospective validation study is required to validate the results. Second, whether all patients were under the same condition before blood collection was uncertain. Third, the results of this study are not suitable for cervical cancer patients after neoadjuvant therapy.

\section{Conclusions}

Nevertheless, this study found that the preoperative SIS is a novel and simple prognostic factor for cervical cancer. In clinical practice, the SIS could be considered as a supplement to the FIGO stage system and assist in the identification of high-risk patients among patients with the same FIGO stage.

\section{Abbreviations}

OS: overall survival; SIS: Systemic Inflammatory Score; Alb, albumin; LMR, lymphocyte-to-monocyte ratio; NLR: neutrophil-to-lymphocyte ratio; PLR: platelet-to-lymphocyte ratio; SCC: squamous cell carcinoma; AC: adenocarcinoma

\section{Declarations}

\section{Acknowledgements}

No acknowledgements.

\section{Author contributions:}

$\mathrm{XM}$ and PMS conceived of the study, analyzed the data, and drafted the manuscript; QBW and LZC helped revise the manuscript critically for important intellectual content; XQS and XYX helped collect data 
and design the study. All authors read and approved the final manuscript.

\section{Funding}

No funding.

\section{Availability of data and materials}

The datasets used and/or analyzed during the current study are available from the corresponding author on reasonable request.

\section{Ethics approval and consent to participate}

This retrospective study was approved by the Ethics Committee of the Fujian Provincial Maternity and Children's Hospital. Written informed consent was obtained from all participants.

\section{Consent for publication}

Not applicable.

\section{Competing interests}

The authors declare that they have no competing interests.

\section{Author details}

${ }^{1}$ Department of Gynecology, Fujian Maternity and Child Health Hospital, Affiliated Hospital of Fujian Medical University, Fuzhou, China

${ }^{2}$ Laboratory of Gynecologic Oncology, Fujian Maternal and Child Health Hospital, Affiliated Hospital of Fujian Medical University, Fuzhou, China

\section{References}

1. Bray F, Ferlay J, Soerjomataram I, Siegel RL, Torre LA, Jemal A. Global cancer statistics 2018 : GLOBOCAN estimates of incidence and mortality worldwide for 36 cancers in 185 countries. CA: a Cancer Journal for Clinicians. 2018;68(6):394-424.

2. Wang W, Zhang F, Hu K, Hou X. Image-guided, intensity-modulated radiation therapy in definitive radiotherapy for 1433 patients with cervical cancer. Gynecol Oncol. 2018;151:444-8.

3. Siegel RL, Miller KD, Jemal A. Cancer statistics. 2016. CA Cancer J Clin. 2016; 66(1): 7-30.

4. Salvatici M, Achilarre MT, Sandri MT, Boveri S, Vanna Z, Landoni F. Squamous cell carcinoma antigen (SCC-Ag) during follow-up of cervical cancer patients: Role in the early diagnosis of recurrence. Gynecol Oncol.2016 07; 142(1): 115-119. 
5. Wang J, Wang T, Yang YY, Chai YY, Shi F, Liu Z. Patient age, tumor appearance and tumor size are risk factors for early recurrence of cervical cancer. Mol Clin Oncol, 2015(3): 363-366.

6. Shinko D, Diakos $\mathrm{Cl}$, Clarke SJ, Charles KA. Cancer-related systemic inflammation: the challenges and therapeutic opportunities for personalized medicine. Clin Pharmacol Ther. 2017;102(4):599610.

7. Galdiero MR, Marone G, Mantovani A. Cancer inflammation and cytokines. Cold Spring Harb Perspect Biol. 2018;10(8):1-17.

8. Kim EY, Lee JW, Yoo HM, Park CH, Song KY. The Platelet-to-Lymphocyte Ratio Versus Neutrophil-toLymphocyte Ratio: Which is Better as a Prognostic Factor in Gastric Cancer? Ann Surg Oncol. 2015;22(13):4363-70.

9. Goto W, Kashiwagi S, Asano Y, Takada K, Takahashi K, Hatano T, et al. Predictive value of lymphocyte-to-monocyte ratio in the preoperative setting for progression of patients with breast cancer. BMC Cancer. 2018;18(1):1137.

10. Lin JP, Lin JX, Cao LL, Zheng CH, Li P, Xie JW, et al. Preoperative lymphocyte-to-monocyte ratio as a strong predictor of survival and recurrence for gastric cancer after radical-intent surgery. Oncotarget. 2017;8(45):79234-47.

11. Miura K, Hamanaka K, Koizumi T, Kitaguchi Y, Terada Y, Nakamura D, et al. Clinical significance of preoperative serum albumin level for prognosis in surgically resected patients with non-small cell lung cancer: Comparative study of normal lung, emphysema, and pulmonary fibrosis. Lung Cancer. 2017;111:88-95.

12. Chang $Y, A n H, X u L$, Zhu Y, Yang Y, Lin Z, et al. Systemic inflammation score predicts postoperative prognosis of patients with clear-cell renal cell carcinoma. Br J Cancer. 2015;113(4):626-33.

13. Suzuki Y, Okabayashi K, Hasegawa H, Tsuruta M, Shigeta K, Kondo T, et al. Comparison of Preoperative Inflammation-based Prognostic Scores in Patients With Colorectal Cancer. Ann Surg. 2018;267(3):527-31.

14. Camp RL, Dolled-Filhart M, Rimm DL. X-tile: a new bio-informatics tool for biomarker assessment and outcome-based cut-point optimization. Clin Cancer Res. 2004;10(21):7252-9.

15. Heagerty PJ, Lumley T, Pepe MS. Time-dependent ROC curves for censored survival data and a diagnostic marker. Biometrics. 2000;56(2):337-44.

16. Balkwill F, Mantovani A. Inflammation and cancer: back to Virchow? Lancet. 2001;357(9255):53945.

17. Shinko D, Diakos $\mathrm{Cl}$, Clarke SJ, Charles KA. Cancerrelated systemic inflammation: the challenges and therapeutic opportunities for personalized medicine. Clin Pharmacol Ther. 2017;102(4):599-610.

18. Huang H, Liu Q, Zhu L, Zhang Y, Lu X, Wu Y, et al. Prognostic Value of preoperative Systemic ImmuneInfammation Index in patients with Cervical Cancer. Sci Rep. 2019;03(1):3284. 9 ).

19. Matsuo K, Hom MS, Moeini A, Machida H, Takeshima N, Roman LD, et al. Significance of monocyte counts on tumor characteristics and survival outcome of women with endometrial cancer. Gynecol Oncol. 2015;138:332-8. 
20. Kwon BS, Jeong DH, Byun JM, Lee TH, Choi KU, Song YJ, et al. Prognostic value of preoperative lymphocyte-monocyte ratio in patients with ovarian clear cell carcinoma. J Cancer. 2018;9(7):112734.

21. Valle-Mendiola A, Gutiérrez-Hoya A, Lagunas-Cruz M, Weiss-Steider B, Soto-Cruz I. Pleiotropic Effects of IL-2 on Cancer: Its Role in Cervical Cancer. Mediators Inflamm. 2016; 2016: 2849523.

22. Anderson KG, Stromnes IM, Greenberg PD. Obstacles Posed by the Tumor Microenvironment to T cell Activity: A Case for Synergistic Therapies. Cancer Cell. 2017;03(13):31(3):311-25.

23. Li J, Wang J, Chen R, Bai Y, Lu X. The prognostic value of tumor-infiltrating T lymphocytes in ovarian cancer. Oncotarget. 2017;8:15621-31.

24. Quigley DA, Kristensen V. Predicting prognosis and therapeutic response from interactions between lymphocytes and tumor cells. Mol Oncol. 2015;9:2054-62.

25. Feng F, Zheng G, Wang Q, Liu S, Liu Z, Xu G, et al. Low lymphocyte count and high monocyte count predicts poor prognosis of gastric cancer. BMC Gastroenterol. 2018;18:148.

26. Diakos $\mathrm{Cl}$, Charles KA, McMillan DC, Clarke SJ. Cancer-related inflammation and treatment effectiveness. Lancet Oncol. 2014;15(11):e493-5-3.

27. Raposo TP, Pires I, Carvalho MI, Prada J, Argyle DJ, Queiroga FL. Tumour-associated macrophages are associated with vascular endothelial growth factor expression in canine mammary tumours. Vet Comp Oncol. 2015 Dec;13(4):464-74.

28. Chen L, Zhang F, Sheng XG, Zhang SQ. Decreased pretreatment lymphocyte/monocyte ratio is associated with poor prognosis in stage Ibl-lla cervical cancer patients who undergo radical surgery. Onco Targets Ther. 2015, (8): 1355-1362.

29. Zheng RR, Huang XW, Liu WY, Lin RR, Zheng FY, Lin F. Nomogram Predicting Overall Survival in Operable Cervical Cancer Patients. Int J Gynecol Cancer. 2017;27:987-93.

\section{Figures}




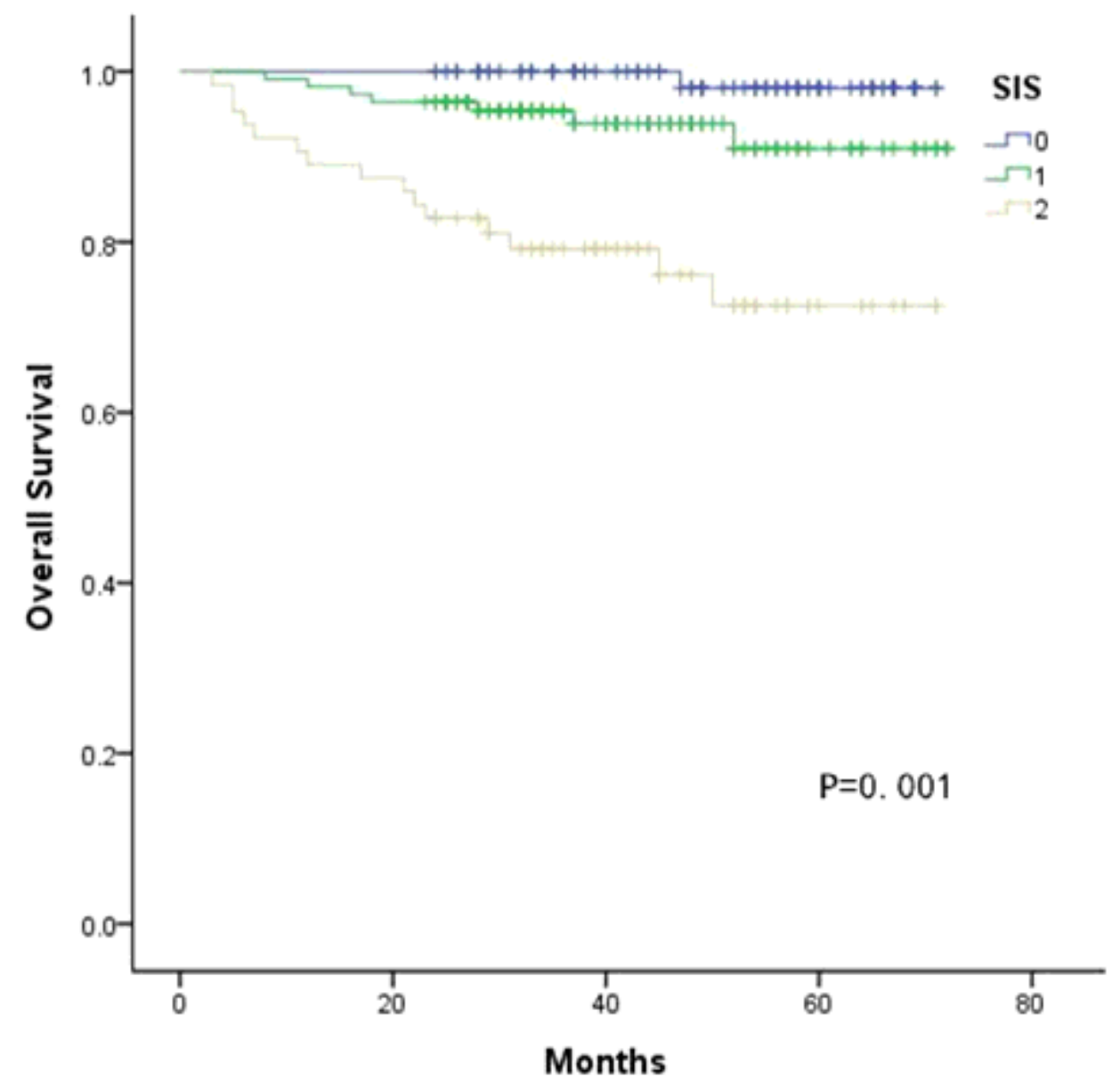

Figure 1

Kaplan-Meier analysis of OS of CC patients according to the SIS.
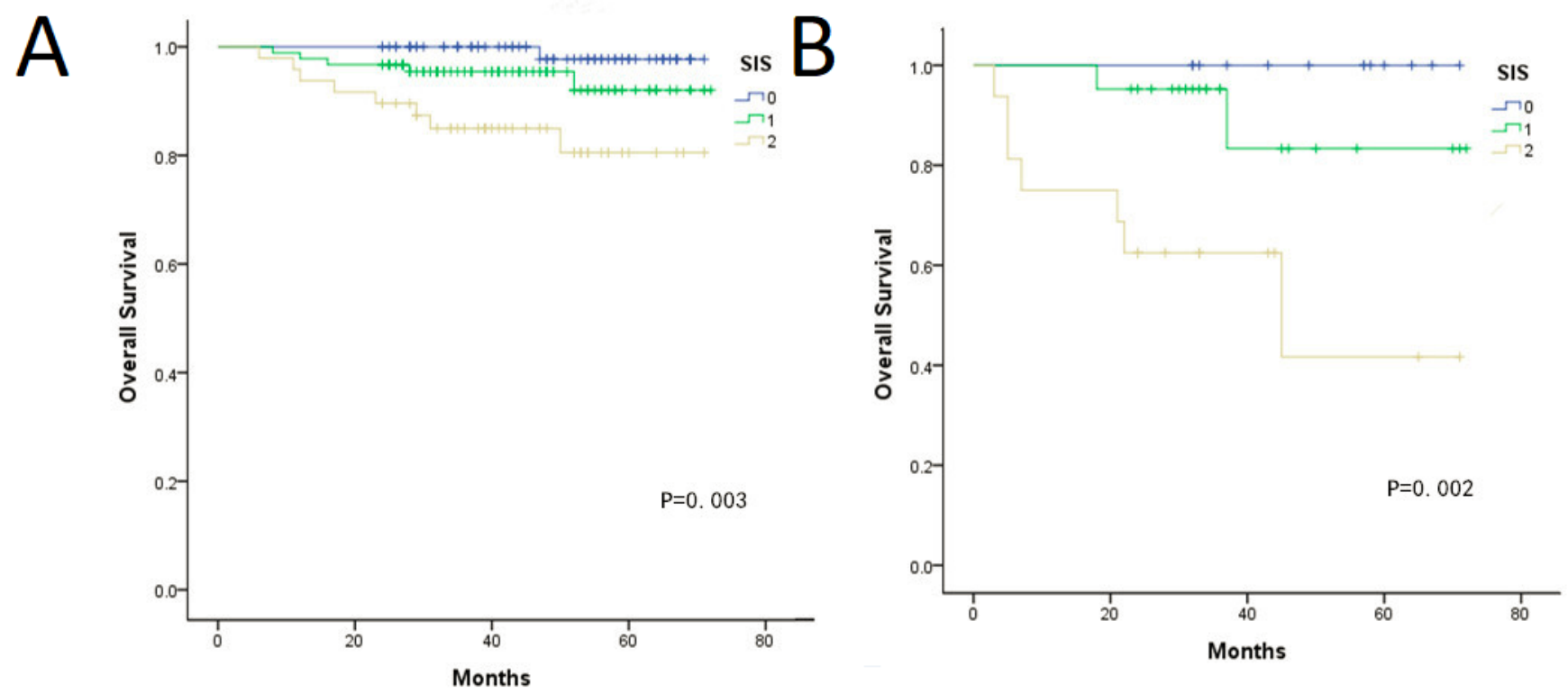

Figure 2 
a Association of the SIS with the OS of patients with FIGO stage lb. b Association of the SIS with the OS of patients with FIGO stage lla.

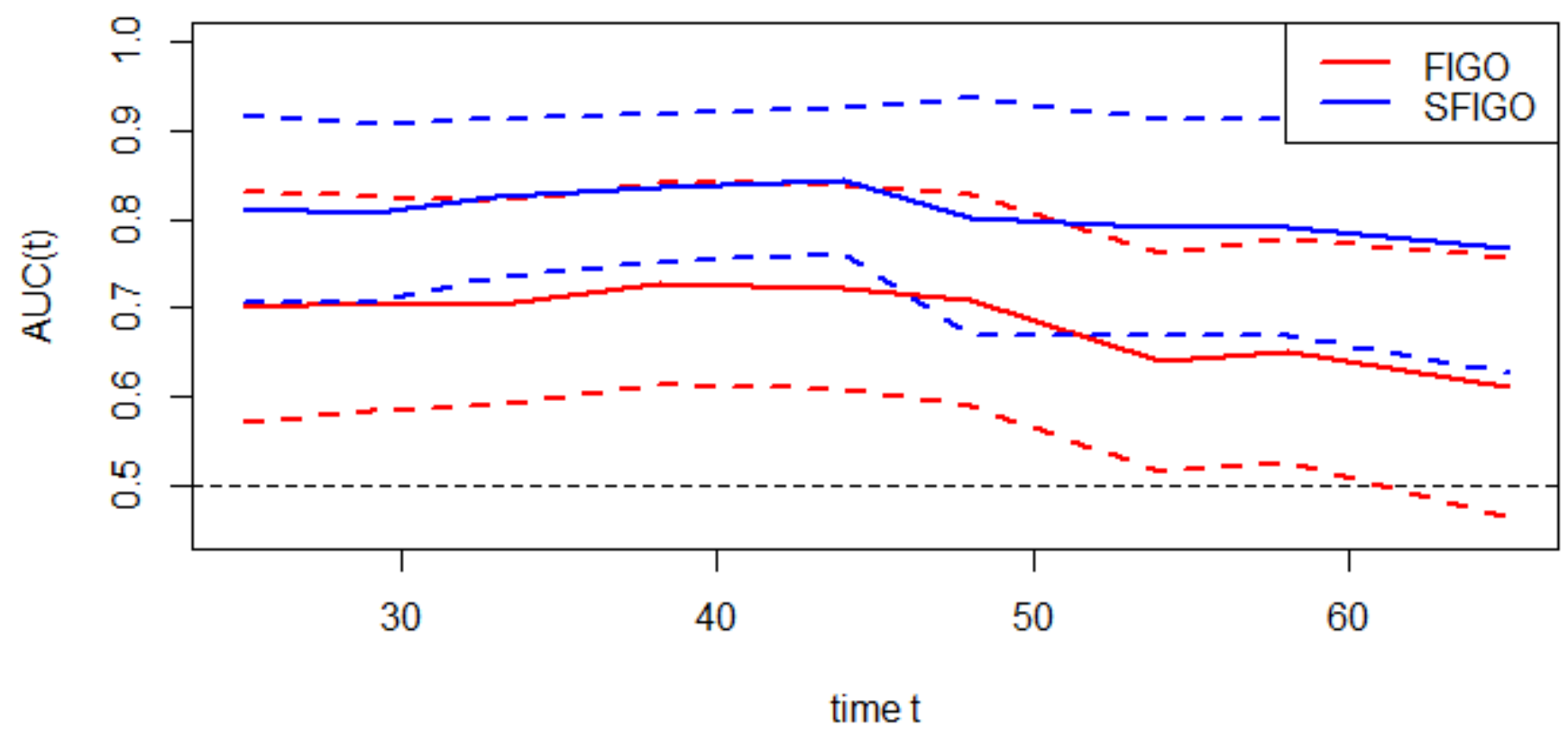

Figure 3

Time-dependent ROC curves of FIGO stage only and new model (FIGO stage and SIS, SFIGO) for the prediction of OS. OS indicates Overall Survival. Supplemental Fig. 1 The cut-off value of LMR according to X-tile. LMR indicates lymphocyte-to-monocyte ratio.

\section{Supplementary Files}

This is a list of supplementary files associated with this preprint. Click to download.

- SupplementalFig.1.tif 